\title{
Low-dose Aspirin for Primary Prevention of Adverse Pregnancy Outcomes in Twin Pregnancies: An Observational Cohort Study Based on Propensity Score Matching
}

\author{
Ying Ye ${ }^{1}$, Li Wen ${ }^{1}$, Xiyao Liu ${ }^{1}$, Lan Wang ${ }^{2}$, Yamin Liu ${ }^{2}$, Richard Saffery ${ }^{3}$, Mark Kilby ${ }^{4}$ \\ Chao Tong ${ }^{1}$, Hongbo Qi ${ }^{1}$, and Philip Baker ${ }^{5}$ \\ ${ }^{1}$ Chongqing Medical University First Affiliated Hospital \\ ${ }^{2}$ Chongqing Health Center for Women and Children \\ ${ }^{3}$ Murdoch Childrens Research Institute \\ ${ }^{4}$ University of Birmingham College of Medical and Dental Sciences \\ ${ }^{5}$ University of Leicester
}

October 15,2020

\begin{abstract}
Abstract Objective: We aimed to preliminarily assess whether low-dose aspirin(LDA)is beneficial in preventing preeclampsia in twin pregnancies. Design: An observational cohort study. Setting: Two hospital centers in Chongqing, China. Population: 932 twin-pregnant women. Methods: Among 932 participants, 277 in the First Affiliated Hospital of Chongqing Medical University were routinely treated with aspirin (100mg daily) from 12-16 weeks to 35 weeks of gestational age, while 655 in Chongqing Health Center for Women and Children were not taking aspirin during the whole pregnancy. We followed each subject and the individual details were recorded. Main Outcome Measures: The main outcome was to compare the incidence of preeclampsia in women with or without aspirin intaking. Results: LDA significantly reduced the risk of PE (OR: 0.48, 95\% CI: 0.24-0.95, $\mathrm{p}=0.048$ ) and $\mathrm{i} 34$ weeks preterm birth (OR: 0.50, 95\% CI: 0.29-0.86, $\mathrm{p}=0.013$ ), but showed possible benefits to lower the rate of SGA babies (OR: 0.74, 95\% CI: 0.55-1.00, p=0.063). Moreover, the risk of postpartum hemorrhage was not increased by LDA (OR: 0.89, 95\% CI: 0.35-2.26, $\mathrm{p}=1.000$ ). Conclusions: Treatment with low-dose aspirin in women pregnant with twins could offer some protection against adverse pregnancy outcomes in the absence of any significantly increased risk of postpartum hemorrhage. Funding: The National Key Research and Development Program of China (2018YFC1002900), and National Natural Science Foundation of China $(81520108013,81771613,81671488,81871189)$. Keywords: Twin pregnancy, low-dose aspirin, preeclampsia, preterm birth, small for gestational age
\end{abstract}

\section{Tweetable abstract}

Low-dose aspirin can reduce the risk of adverse outcomes in twin pregnancies.

\section{Introduction}

The prevalence of twin pregnancy is increasing, primarily as a result of the use of Assisted Reproductive Technologies (ART). Relative to singletons, twin pregnancies are associated with increased maternal and perinatal mortality and morbidity, the associated perinatal adverse outcomes, including preeclampsia (PE), small for gestational age (SGA) babies, and preterm birth (PTB). ${ }^{1}$ Recently, it has been reiterated that the incidence of $\mathrm{PE}$ in twin pregnancies is 3-4 times folds higher compared to singletons. ${ }^{2} \mathrm{~A}$ clinical cohort study containing 321 twin pregnancies demonstrated the incidence of at least one twin with birthweight $<10^{\text {th }}$ centile was $47 \%$, while at least one with birthweight $<5^{\text {th }}$ centile was $27 \%{ }^{3}$ It is also reported that the percentage of PTB in twin pregnancies is significantly higher than singletons, with $50-60 \%$ delivering 
prior to 37 weeks. ${ }^{4}$ The increasing morbidity and mortality associated with adverse pregnancy outcomes of PTB, PE, and SGA remain major concerns internationally. Although the etiology of these complications has yet been fully elucidated, their development is generally associated with increased inflammation and/or hypoxia. ${ }^{5-8}$

The well-described anti-inflammatory and anti-coagulation properties of aspirin have made it an appealing target for preventing gestational hypertensive disorders, but the specific mechanism is still unclear. ${ }^{9}$ According to recent studies, we have a better understanding of the effects of aspirin on the prophylaxis of PE, PTB, and SGA. ${ }^{10-12}$ Based on current data, the American College of Obstetricians and Gynecologists (ACOG) recommends daily low-dose aspirin $(81 \mathrm{mg} /$ day) in pregnancy to prevent adverse outcomes and while not increasing the risk of postpartum hemorrhage. ${ }^{13}$ But another meta-analysis ${ }^{14}$ concluded that a dose of aspirin between 100-150mg was more beneficial, especially if initiated before 16 weeks. However, the optimal dose has not reached a consensus. Unlike in singletons, few studies have explored the potential beneficial (or otherwise) effects of aspirin in twin pregnancies. The ACOG-guideline recommended pregnant women to take aspirin based on circumstantial evidence - according to the incidence of PE was much higher in twin pregnancies. ${ }^{13}$ But there is a relative lack of direct evidence whether aspirin could reduce these adverse outcomes in twin pregnancies.

Given the limited data on twin pregnancies and the often conflicting results in singleton pregnancies, we undertook an observational cohort study based on real-world data. We aimed to explore the potential benefits of aspirin on twin pregnancies and provides a theoretical basis for further researches.

\section{Methods}

\section{Study design and participants}

This study was designed as an observational cohort based on real-world data in two centers from March 2016 to December 2018. Pregnant women with twins who 'booked' at the First Affiliated Hospital of Chongqing Medical University were routinely offered prophylaxis with low-dose aspirin (LDA group) before 16 weeks of gestational age and those recruited from Chongqing Health Center for Women and Children were not required to take aspirin and regarded as the control group (NC group). This difference was based upon clinician preference at the two institutions. In the LDA group, participants were administered aspirin from the day when registered. Aspirin was taken at the dose of 100mg per night from recruitment until 35 weeks of gestation age. Inclusion criteria were: Twin pregnancies were diagnosed by ultrasound in the first visit. Those who did not start their first antenatal care in the two centers but delivered in these 2 hospitals were excluded in the final analysis due to incomplete records of prior medications. Pregnant women were asked to have a visit every 2 weeks before 28 weeks of gestational age, and every 1 week when the gestational week was over 28 .

\section{Data collection}

All pregnant patient clinical, demographic, neonatal assessments, and outcomes data during pregnancy and at delivery were recorded in the Case Report Form (CRF). All the information was concurrently recorded in both Hospital Information Systems (HIS) and Cohort Management Systems. According to the medical records, each participant in the LDA group was confirmed to have an over $90 \%$ drug compliance.

\section{Statement about aspirin}

Due to the lack of consensus in using LDA for twin pregnancies, the two centers participated in this study carried out different policies on aspirin use for twin pregnancies and thus made this observational study feasible. Given that the specification of aspirin tablets in China is either 25mg or 100mg, and ACOG recommendation for pregnant women is $81 \mathrm{mg}$ daily, therefore, $100 \mathrm{mg} / \mathrm{d}$ was prescribed to participants to avoid under dosage.

\section{Outcome Measures}


The primary outcome was the incidence of PE and the secondary outcomes included the gestation of birth (allowing assessment of preterm birth) and birth weight (allowing assessment of the fetus is small for gestational age). The clinical safety of aspirin was evaluated by the occurrence of antenatal or postpartum hemorrhage. PE was defined by evaluated blood pressure (blood pressure[?]140/90 $\mathrm{mmHg}$ associated with proteinuria (>300 mg/day) or blood pressure[?]160/110mmHg alone) after 20 weeks of gestational age. ${ }^{13,} 15$ Blood pressure was measured by a mercury sphygmomanometer and urine samples were collected and tested by the clinical laboratory in each hospital. Once diagnosed, patients would be treated as standard treatment guidelines. The fetus being small for gestational age (SGA) was defined as the birth weight of either twin was below the $10^{\text {th }}$ percentile of the average in the same gestational age. PTB was regarded as delivering before 34 weeks 0 days for twin pregnancies. ${ }^{16,} 17$ When calculating the amount of bleeding, intraoperative blood loss was recorded by the container of suction apparatus, while postoperative bleeding volume was counted by the weighing method. Cesarean postpartum hemorrhage was defined as postoperative bleeding volume more than $1000 \mathrm{ml}$ in $24 \mathrm{~h}$. These diagnostic criteria were unified in two centers before the cohort study was initiated.

\section{Statistical analysis}

The study was initially designed to detect an $8 \%$ absolute difference in PE rate with $80 \%$ power and a $5 \%$ type I error rate. ${ }^{18}$ We assume the incidence of PE was $4 \%$ in the LDA group, while in the control group the rate was $12 \%$. Accounting for a potential $20 \%$ loss to follow-up, therefore, a sample size of 424 (212 in the LDA group, 212 in the Control group) was needed. However, the actual loss to follow-up (including 47 subjects met twin-to-twin transfusion syndrome) of $12.90 \%(\mathrm{n}=138)$ was less than the $20 \%$ expected and we finally followed up 932 patients (277 in the LDA group and 655 in the control group).

To adjust the unmatched baseline characteristics, a 1:1 propensity score matching (PSM) was applied between the LDA group and the control group to simulate a randomized controlled trial (RCT) of the variables. The matching algorithm was the nearest-neighbor matching and the caliper was set at 0.1 . The standardized mean difference (SMD) was calculated by using R (version 2.15.3, the R Foundation for Statistical Computing, Tsinghua University, China; downloaded at http://www.R-project.org/). The value of SMD[?]0.1 was considered to be a negligible difference. We also performed a sensitivity analysis to evaluate the biases caused by propensity score matching. SPSS version 22.0 software (SPSS Inc, Chicago, USA) was used for all the statistical analyses.

We dealt with data via an independent t-test and described as mean \pm standard deviation if variables were in accordance with a normal distribution. Otherwise, variables were described as mean \pm quartile and examined by the Kruskal-Wallis test. Differences in the classified variables were presented by percentage and evaluated by the McNemar's test. $P<0.05$ or $\mathrm{SMD}_{\mathfrak{i} 0.1}$ was considered as significant. A conditional logistic regression model was used in paired data (after PSM), while an unconditional logistic model was applied in the sensitivity analysis. Confounding factors including age, BMI, and those which were previously reported to be connected with preeclampsia or were unmatched between LDA and normal groups.

\section{Funding}

This work was supported by the National Key Research and Development Program of China (2018YFC1002900), and the National Natural Science Foundation of China (81520108013, 81771613, 81671488, 81871189). The funders had no involvement in the study design, data collection and analysis, interpretation of data, and preparation of the manuscript. The corresponding authors had full access to all of the data in the study and had the final responsibility to submit for publication.

\section{Results}

\section{Baseline Characteristics}

In total, 1070 twin pregnancies were recruited in this study, 138 were excluded for final analysis. Among them, 91 subjects were lost to follow-up and 47 were diagnosed with Twin to twin transfusion syndrome (TTTs) and underwent a medical intervention. Finally, we obtained the completed information from 932 
pregnancies (277 in the LDA group, 655 in the control group). (Figure 1) Before the PSM, the baseline characteristics vary widely. Ages of mothers in the LDA group (29.55y) were younger than that in the control group $(30.22 \mathrm{y}, p=0.022)$. In the LDA group, the percentage of spontaneous conception was $65.3 \%$, which was higher than the control group $(36.9 \%, p<0.001)$. Chorionicity subtypes were also quite different between the two groups $(p<0.001)$. These data were shown in Table.1.

After PSM, 235 women who received LDA treatment and 235 who did not use LDA were finally paired for analysis. The characteristics were matched well (all $p>0.05 \&$ all $\mathrm{SMD}<0.1$ ). These data were shown in Table.2.

\section{Main Outcomes}

In this observational cohort study (after PSM), the incidence of PE was $4.68 \%$ in the LDA group, while in the control group (vs. 9.79\%), and LDA showed a significant reduction of PE (OR:0.48, 95\% CI: 0.24-0.95, $p$ $=0.048$ ). While the risk of gestational hypertension was not reduced by LDA (OR:0.88, 95\% CI: 0.45-1.73, $p$ $=0.855$ ). The median of the gestational length of the pregnancy (in days) was larger in the LDA group than in controls (259 vs. 254,p ¡0.001). Moreover, the occurrence of PTB was also significantly reduced in the LDA group compare to the control group (7.66\% vs.15.32\%, OR: 0.50, 95\% CI:0.29-0.86, $p=0.013)$. Notably, LDA significantly lowered the risk of spontaneous preterm birth, not for iatrogenic. However, the protective role of LDA on SGA was not strong ( $23.40 \%$ vs. $31.49 \%$, OR:0.74, 95\% CI: $0.55-1.00, p=0.063$ ), even calculated in selective SGA (sSGA) subgroups (20.00\% vs. $27.80 \%$, OR:0.72, 95\% CI: $0.51-1.01, p=0.060)$. Considering the coagulation function might be impaired by aspirin administration, bleeding volume during delivery was recorded, which was $505.66+-300.96 \mathrm{ml}$ and $524.57+-445.61 \mathrm{ml}$ in the LDA group and Control group, respectively $(p=0.590)$. Furthermore, the incidences of cesarean postpartum hemorrhage in the LDA group and control group were $3.40 \%$ and $3.84 \%$ respectively (OR:0.89, 95\% CI: $0.35-2.26, p=1.000$ ). (Table $3)$.

\section{Sensitivity analysis}

We performed a sensitivity analysis to evaluate the possible selective biases made by PSM and the multivariate non-conditional logistics regression was employed. The main pregnancy outcomes mentioned before were analyzed respectively using logistics-regression to evaluate the efficacy of aspirin. The independent variables including aspirin-intake, age, BMI, BMI increase, gravidity, Chorionic types, and fertilization methods.

As shown in supplementary tables, the logistic regression models were made for each outcome. The protective role of LDA on PE and PTB is undoubted, for the identical conclusions between the two methods. However, the effect of LDA on SGA was uncertain as to the disparate outcomes before (AOR: 0.76, 95\%CI: 0.45-0.91) and after PSM (OR:0.74, 95\%CI: 0.55-1.00). In the whole-data regression model, no matter which variable was removed, the results still kept the same as before, and so did in the regression model after PSM. That's to say, the discrepancy was not caused by single variables. When PSM was performed, 42 and 420 subjects were excluded in the LDA and control group. Among them, the percentage of SGA was a little more in the control group $(23.8 \%$ vs. $27.4 \%, p=0.717)$, and this may make some minor difference in the conclusion. (Table S1-S5)

According to sensitivity analysis, we can conclude no obvious biases were made by PSM, and we can confirm LDA as a protector to PE and PTB. For SGA, we still considered it could also provide some benefits against SGA, and this point needs further studies to confirm.

\section{Discussion}

\section{Main Findings}

Overall, this observational cohort study indicates that the early pregnancy prophylaxis with aspirin was associated with lower rates of PE and PTB; whilst not associated with an increased risk of postpartum 
hemorrhage in twin pregnancies. Our data appear to demonstrate that LDA prophylaxis also reduces the risk of SGA.

\section{Interpretations}

$\mathrm{PE}$ is defined as hypertension arising after 20 weeks of gestational age with proteinuria or other signs of end-organ damage and is a significant cause of maternal and perinatal morbidity and mortality, particularly when of early-onset. The basic pathophysiology of the condition is still poorly understood but there is evidence that poor trophoblast invasion of the decidua-myometrium with the suboptimal remodeling of the maternal spiral arteries and an imbalance of angiogenic / anti-angiogenic proteins leading to an inflammatory response, endothelium dysfunction, increased platelet aggregation predisposing to thrombosis and placental infarcts. Aspirin, at a daily dose as low as $60 \mathrm{mg}$, selectively and irreversibly inactivates the cyclooxygenase-1 enzyme, suppressing the production of prostaglandins and thromboxane and inhibiting inflammation and platelet aggregation in pregnancy. ${ }^{19}$ Subsequent to this finding, large meta-analyses and systematic reviews have consistently shown LDA to be effective in reducing the incidence of $\mathrm{PE},{ }^{20-24}$ the benefits are generally modest and ideal candidates to receive LDA are not well-defined. Recently, the Aspirin for Evidence-based Preeclampsia Prevention (ASPRE) trial (in singleton pregnancy) has demonstrated that aspirin at a daily dose of $150 \mathrm{mg}$, initiated before 16 weeks of gestational age, and given at night to a high-risk population, identified by a combined first-trimester screening test, reduces the incidence of preterm pre-eclampsia by $62 \% .{ }^{25}$ A secondary analysis of the ASPRE trial data also demonstrated a reduction in the length of stay in the neonatal intensive care unit by $68 \%$, compared with placebo, mainly due to a reduction in births before 32 weeks of gestational age with preeclampsia. ${ }^{26}$

Different national guidelines have varying recommendations for low dose aspirin prophylaxis in twin pregnancy. The American College of Obstetrician \& Gynecologist recommendations ${ }^{13}$ recommends low dose aspirin ( $81 \mathrm{mg}$ /day) initiated between 12 weeks and 28 weeks (optimally before 16 weeks) until delivery, in multifetal gestations (as a high-risk factor). The NICE Guidelines published in the $\mathrm{UK}^{27}$ recommends prophylactic low dose aspirin $(75-150 \mathrm{mg} /$ day $)$ from 12 weeks until the birth of the baby if it is a first pregnancy, the mother is 40 years or older, has a pregnancy interval of 10 years, has a body mass index of $>$ $35 \mathrm{~kg} / \mathrm{m}^{2}$ or a family history of pre-eclampsia in twin or triplet pregnancies. More recently, A prospective study in twin pregnancy randomizing women to $100 \mathrm{mg}$ /day aspirin versus placebo before 16 weeks noted an overall reduction in pre-eclampsia from $16 \%$ to $6 \%$ respectively (OR $0.32,95 \%$ CI $0.12-0.82$ ) (a result that was increased in the cohort of twin pregnancies with high hCG levels (threshold of $29.96 \mathrm{iu} / \mathrm{mL}))^{28}$

The efficacy of LDA for prophylaxis against the development of PE in singletons is proven, especially in 'high-risk pregnancies', but the underlying mechanisms of action are unknown. As we know, PE was mainly induced by ischemia-hypoxia of the placenta. Soluble fms-like tyrosine kinase 1 (sFlt-1) would be released by trophoblast cells when the environment was anoxic, and ischemia-hypoxia would be aggravated by high levels of sFlt-1. Recently, several studies focused on the mechanism of aspirin acting on trophoblast cells. The release of sFlt-1 could be inhibited by aspirin through diverse pathways, and the ischemia-hypoxia of the placenta would be alleviated. ${ }^{29-32}$ It has been fully reported that the average sFlt- 1 level and the sFlt-1/PlGF ratio were higher in twin pregnancies than singleton pregnancies and indicated that the more impairment to maternal vascular functions. ${ }^{33-35}$ Thus, for twin pregnancies, it is more essential to protect maternal vessels. The mechanism of aspirin mentioned above provides bases for the treatment of PE and SGA, but its efficacy in multiple gestations was still uncertain because of poor clinical evidence. A meta-analysis contained 6 RCTs and 898 pregnancies have shown that using LDA can significantly reduce the risk of PE in multiple gestations but it concluded the evidence was at low levels because of the limitation of each RCT. ${ }^{36}$ The occurrence of PE in our study was lower in LDA-treated mothers and the results were consistent with Euser et al. $^{28}$

Whether the usage of aspirin could reduce SGA has been a matter of debate. ${ }^{36-38}$ Bergeron et al ${ }^{36}$ focused on multiple gestations, concluded that the risk of SGA was not decreased by LDA (RR:1.09, 95\% CI: 0.80-1.47). But in another meta-analysis for singletons, the early initiated LDA could reduce the odds of SGA (RR:0.47, 95\% CI: 0.30-0.74). ${ }^{37}$ However, our data only presented a possible reduction of SGA by using LDA and this 
conclusion still needs further studies.

The onset of and PTB remains multifactorial with infection or inflammation, uterine overdistention, or endocrine and immunological disorders. ${ }^{39-41}$ Ischemia of placenta and vascular disorders have also been shown to contribute to the pathogenesis of PTB. ${ }^{42,}{ }^{43}$ Aspirin could down-regulate many inflammatory factors, and improve blood supply in the placenta; thus, aspirin could theoretically lower the incidence of PTB. And in clinical practice, aspirin showed strong benefits in singletons preventing PTB. ${ }^{10,44,45}$ In Andrikopoulou's study, they found that LDA was associated with a decrease only in $<34 \mathrm{w}$ PTB in singletons. ${ }^{10}$ Another RCT finished by Allshouse et al. investigated aspirin did not work in preventing PPROM or preterm birth in singletons. ${ }^{44}$ A recent double-blind RCT has proved LDA could reduce the risk of PTB and $<34 \mathrm{w}$ PTB. ${ }^{45}$ Although the evidence in singletons was strong, there was not sufficient evidence about LDA in protecting PTB in twins. Our findings suggested that LDA could decrease the occurrence of preterm birth, which was accorded with singleton RCT trials.

\section{Strengths and Limitations}

Our research has several strengths. It's an observational cohort study across two centers and has a longitudinal follow-up for each participant. As a twin pregnant cohort, we have a large number of subjects and owned the whole information of each mother from registration to delivery. We applied PSM and sensitivity analysis to deal with unmatched baseline characteristics, so the conclusions were reliable. To our knowledge, this is the first cohort trial to demonstrate that early pregnancy-initiated LDA could improve the adverse outcomes in twin pregnancies.

As a cohort study based on real-world data, there were some inherent defects. No random was applied in this study, and the baseline characteristics in primary data were not matched, although PSM was applied, we regrettably abandoned a lot of patients for final analysis. Secondary, it is a purely observational study, and usage of aspirin was uncontrolled but uniformed in one medical center. Another shortage of our study was that we just gathered the medical details of patients while other baseline information such as educational background, annual income, and so on were not collected.

Combining with these defects, further multi-center RCTs from multiple centers should be taken into considerations.

\section{Conclusion}

Low-dose aspirin initiated at early gestational age could significantly reduce the risk of adverse outcomes, especially for pre-eclampsia and preterm birth.

\section{Disclosure of interests}

None declared. Completed disclosure of interest forms are available to view online as supporting information.

\section{Contribution to authorship}

HQ, CT, and RS contributed to the study design. YY, LW, YL and LW collected data. YY and XL analyzed data. YY and LW wrote the manuscript. MK contributed to the interpretation of results. PB and $\mathrm{RS}$ assisted with the detailed editing of the manuscript to get it to the appropriate language. HQ and CT revised the final version and are guarantors of this manuscript. All authors made substantial contributions to the paper and read and approved the final manuscript.

\section{Details of ethics approval}

Neither patients nor the public was involved in the study design, analysis of data, or interpretation of study results. This study was approved by the institutional ethics committee of the First Affiliated Hospital of Chongqing Medical University on 29 February 2016 (Project NO.201530), and the informed consent was completed before cohort enrollment.

\section{Funding}


The National Key Research and Development Program of China (2018YFC1002900), and National Natural Science Foundation of China (81520108013, 81771613, 81671488, 81871189 and 82071675).

\section{Acknowledgments}

We would like to acknowledge the support from the "111 program" of the Ministry of Education P.R.C and the State Administration of Foreign Experts Affairs P.R.C., and Key Laboratory of Birth Defects and Reproductive Health of National Health and Family Planning Commission P.R.C.

\section{Data sharing}

The data used and analyzed during the current study are available from the corresponding author on reasonable request.

\section{References}

1. Shavit M, Miller N, Schreiber H, Asali A, Ravid D, Harlev A, et al. Twin pregnancies and perinatal outcomes: a comparison between fresh and frozen embryo transfer: a two-centre study. Reprod Biomed Online. 2019 Feb;38(2):241-8.

2. Francisco C, Wright D, Benko Z, Syngelaki A, Nicolaides KH. Hidden high rate of pre-eclampsia in twin compared with singleton pregnancy. Ultrasound Obstet Gynecol. 2017 Jul;50(1):88-92.

3. Fox NS, Rebarber A, Klauser CK, Roman AS, Saltzman DH. Intrauterine growth restriction in twin pregnancies: incidence and associated risk factors. Am J Perinatol. 2011 Apr;28(4):267-72.

4. Vasak B, Verhagen JJ, Koenen SV, Koster MP, de Reu PA, Franx A, et al. Lower perinatal mortality in preterm born twins than in singletons: a nationwide study from The Netherlands. Am J Obstet Gynecol. 2017 Feb;216(2):161 e1- e9.

5. Kim CJ, Romero R, Chaemsaithong P, Kim JS. Chronic inflammation of the placenta: definition, classification, pathogenesis, and clinical significance. Am J Obstet Gynecol. 2015 Oct;213(4 Suppl):S53-69.

6. Nadeau-Vallee M, Obari D, Quiniou C, Lubell WD, Olson DM, Girard S, et al. A critical role of interleukin-1 in preterm labor. Cytokine Growth Factor Rev. 2016 Apr;28:37-51.

7. Gomez-Lopez N, Motomura K, Miller D, Garcia-Flores V, Galaz J, Romero R. Inflammasomes: Their Role in Normal and Complicated Pregnancies. Journal of immunology. 2019 Dec 1;203(11):2757-69.

8. Rana S, Lemoine E, Granger JP, Karumanchi SA. Preeclampsia: Pathophysiology, Challenges, and Perspectives. Circ Res. 2019 Mar 29;124(7):1094-112.

9. Roberge S, Nicolaides K, Demers S, Villa P, Bujold E. Prevention of perinatal death and adverse perinatal outcome using low-dose aspirin: a meta-analysis. Ultrasound in obstetrics \& gynecology : the official journal of the International Society of Ultrasound in Obstetrics and Gynecology. 2013;41(5):491-9.

10. Andrikopoulou M, Purisch SE, Handal-Orefice R, Gyamfi-Bannerman C. Low-dose aspirin is associated with reduced spontaneous preterm birth in nulliparous women. Am J Obstet Gynecol. 2018 Oct;219(4):399 e1- e6.

11. Meher S, Duley L, Hunter K, Askie L. Antiplatelet therapy before or after 16 weeks' gestation for preventing preeclampsia: an individual participant data meta-analysis. Am J Obstet Gynecol. 2017 Feb;216(2):121-8 $\mathrm{e} 2$.

12. Loussert L, Vidal F, Parant O, Hamdi SM, Vayssiere C, Guerby P. Aspirin for prevention of preeclampsia and fetal growth restriction. Prenat Diagn. 2020 Apr;40(5):519-27.

13. ACOG Committee Opinion No. 743: Low-Dose Aspirin Use During Pregnancy. Obstetrics and gynecology. 2018;132(1):e44-e52. 
14. Roberge S, Bujold E, Nicolaides K. Aspirin for the prevention of preterm and term preeclampsia: systematic review and metaanalysis. American journal of obstetrics and gynecology. 2018;218(3):287-93.e1.

15. Mol BWJ, Roberts CT, Thangaratinam S, Magee LA, de Groot CJM, Hofmeyr GJ. Pre-eclampsia. Lancet. 2016 Mar 5;387(10022):999-1011.

16. Rosen H, Stratulat V, Aviram A, Melamed N, Barrett J, Glanc P. Mid-trimester cervical consistency index measurement and prediction of preterm birth before 34 and 37 weeks in twin pregnancy. Ultrasound Obstet Gynecol. 2020 Oct;56(4):626-8.

17. Menzies R, Li ALK, Melamed N, Shah PS, Horn D, Barrett J, et al. Risk of singleton preterm birth after prior twin preterm birth: a systematic review and meta-analysis. Am J Obstet Gynecol. 2020 Aug;223(2):204 e1- e8.

18. Chow S. Sample size calculations in clinical research: Taylor \& Francis.

19. Louden K, Broughton Pipkin F, Symonds E, Tuohy P, O'Callaghan C, Heptinstall S, et al. A randomized placebo-controlled study of the effect of low dose aspirin on platelet reactivity and serum thromboxane B2 production in non-pregnant women, in normal pregnancy, and in gestational hypertension. British journal of obstetrics and gynaecology. 1992;99(5):371-6.

20. Askie L, Duley L, Henderson-Smart D, Stewart L. Antiplatelet agents for prevention of pre-eclampsia: a meta-analysis of individual patient data. Lancet (London, England). 2007;369(9575):1791-8.

21. Coomarasamy A, Honest H, Papaioannou S, Gee H, Khan K. Aspirin for prevention of preeclampsia in women with historical risk factors: a systematic review. Obstetrics and gynecology. 2003;101(6):1319-32.

22. LeFevre M. Low-dose aspirin use for the prevention of morbidity and mortality from preeclampsia: U.S. Preventive Services Task Force recommendation statement. Annals of internal medicine. 2014;161(11):81926.

23. Bujold E, Roberge S, Lacasse Y, Bureau M, Audibert F, Marcoux S, et al. Prevention of preeclampsia and intrauterine growth restriction with aspirin started in early pregnancy: a meta-analysis. Obstetrics and gynecology. 2010;116:402-14.

24. Henderson J, Whitlock E, O'Connor E, Senger C, Thompson J, Rowland M. Low-dose aspirin for prevention of morbidity and mortality from preeclampsia: a systematic evidence review for the U.S. Preventive Services Task Force. Annals of internal medicine. 2014;160(10):695-703.

25. Rolnik D, Wright D, Poon L, Syngelaki A, O'Gorman N, de Paco Matallana C, et al. ASPRE trial: performance of screening for preterm pre-eclampsia. Ultrasound in obstetrics \& gynecology : the official journal of the International Society of Ultrasound in Obstetrics and Gynecology. 2017;50(4):492-5.

26. Wright D, Rolnik D, Syngelaki A, de Paco Matallana C, Machuca M, de Alvarado M, et al. Aspirin for Evidence-Based Preeclampsia Prevention trial: effect of aspirin on length of stay in the neonatal intensive care unit. American journal of obstetrics and gynecology. 2018;218(6):612.e1-.e6.

27. National Guideline A. National Institute for Health and Care Excellence: Clinical Guidelines. Twin and Triplet Pregnancy. London: National Institute for Health and Care Excellence (UK)

Copyright (C) NICE 2019.; 2019.

28. Euser AG, Metz TD, Allshouse AA, Heyborne KD. Low-dose aspirin for pre-eclampsia prevention in twins with elevated human chorionic gonadotropin. J Perinatol. 2016 Aug;36(8):601-5.

29. Atallah A, Lecarpentier E, Goffinet F, Doret-Dion M, Gaucherand P, Tsatsaris V. Aspirin for Prevention of Preeclampsia. Drugs. 2017 Nov;77(17):1819-31. 
30. Din FV, Valanciute A, Houde VP, Zibrova D, Green KA, Sakamoto K, et al. Aspirin inhibits mTOR signaling, activates AMP-activated protein kinase, and induces autophagy in colorectal cancer cells. Gastroenterology. 2012 Jun;142(7):1504-15 e3.

31. Li C, Raikwar NS, Santillan MK, Santillan DA, Thomas CP. Aspirin inhibits expression of sFLT1 from human cytotrophoblasts induced by hypoxia, via cyclo-oxygenase 1. Placenta. 2015 Apr;36(4):446-53.

32. Su MT, Wang CY, Tsai PY, Chen TY, Tsai HL, Kuo PL. Aspirin enhances trophoblast invasion and represses soluble fms-like tyrosine kinase 1 production: a putative mechanism for preventing preeclampsia. J Hypertens. 2019 Dec;37(12):2461-9.

33. Dröge L, Herraìz I, Zeisler H, Schlembach D, Stepan H, Küssel L, et al. Maternal serum sFlt-1/PlGF ratio in twin pregnancies with and without pre-eclampsia in comparison with singleton pregnancies. Ultrasound in obstetrics \& gynecology : the official journal of the International Society of Ultrasound in Obstetrics and Gynecology. 2015;45(3):286-93.

34. Saleh L, Tahitu S, Danser A, van den Meiracker A, Visser W. The predictive value of the sFlt-1/PlGF ratio on short-term absence of preeclampsia and maternal and fetal or neonatal complications in twin pregnancies. Pregnancy hypertension. 2018;14:222-7.

35. Adank M, Broere-Brown Z, Gonçalves R, Ikram M, Jaddoe V, Steegers E, et al. Maternal cardiovascular adaptation to twin pregnancy: a population-based prospective cohort study. BMC pregnancy and childbirth. 2020;20(1):327.

36. Bergeron TS, Roberge S, Carpentier C, Sibai B, McCaw-Binns A, Bujold E. Prevention of Preeclampsia with Aspirin in Multiple Gestations: A Systematic Review and Meta-analysis. Am J Perinatol. 2016 May;33(6):605-10.

37. Bujold E, Roberge S, Lacasse Y, Bureau M, Audibert F, Marcoux S, et al. Prevention of preeclampsia and intrauterine growth restriction with aspirin started in early pregnancy: a meta-analysis. Obstet Gynecol. 2010 Aug;116(2 Pt 1):402-14.

38. Henderson JT, Whitlock EP, O'Connor E, Senger CA, Thompson JH, Rowland MG. Low-dose aspirin for prevention of morbidity and mortality from preeclampsia: a systematic evidence review for the U.S. Preventive Services Task Force. Ann Intern Med. 2014 May 20;160(10):695-703.

39. Goldenberg RL, Culhane JF, Iams JD, Romero R. Epidemiology and causes of preterm birth. Lancet. 2008 Jan 5;371(9606):75-84.

40. Arias F, Rodriquez L, Rayne SC, Kraus FT. Maternal placental vasculopathy and infection: two distinct subgroups among patients with preterm labor and preterm ruptured membranes. Am J Obstet Gynecol. 1993 Feb;168(2):585-91.

41. Romero R, Dey SK, Fisher SJ. Preterm labor: one syndrome, many causes. Science. 2014 Aug $15 ; 345(6198): 760-5$.

42. van Vliet EO, Askie LA, Mol BW, Oudijk MA. Antiplatelet Agents and the Prevention of Spontaneous Preterm Birth: A Systematic Review and Meta-analysis. Obstet Gynecol. 2017 Feb;129(2):327-36.

43. Martin A, Faes C, Debevec T, Rytz C, Millet G, Pialoux V. Preterm birth and oxidative stress: Effects of acute physical exercise and hypoxia physiological responses. Redox Biol. 2018 Jul;17:315-22.

44. Allshouse AA, Jessel RH, Heyborne KD. The impact of low-dose aspirin on preterm birth: secondary analysis of a randomized controlled trial. J Perinatol. 2016 Jun;36(6):427-31.

45. Hoffman MK, Goudar SS, Kodkany BS, Metgud M, Somannavar M, Okitawutshu J, et al. Low-dose aspirin for the prevention of preterm delivery in nulliparous women with a singleton pregnancy (ASPIRIN): a randomised, double-blind, placebo-controlled trial. Lancet. 2020 Jan 25;395(10220):285-93. 


\section{Hosted file}

Table 1.pdf available at https://authorea.com/users/367407/articles/486849-low-doseaspirin-for-primary-prevention-of-adverse-pregnancy-outcomes-in-twin-pregnancies-anobservational-cohort-study-based-on-propensity-score-matching

\section{Hosted file}

Table 2.pdf available at https://authorea.com/users/367407/articles/486849-low-doseaspirin-for-primary-prevention-of-adverse-pregnancy-outcomes-in-twin-pregnancies-anobservational-cohort-study-based-on-propensity-score-matching

\section{Hosted file}

Table 3.pdf available at https://authorea.com/users/367407/articles/486849-low-doseaspirin-for-primary-prevention-of-adverse-pregnancy-outcomes-in-twin-pregnancies-anobservational-cohort-study-based-on-propensity-score-matching

\section{Hosted file}

Supplementary tables.pdf available at https://authorea.com/users/367407/articles/486849-1owdose-aspirin-for-primary-prevention-of-adverse-pregnancy-outcomes-in-twin-pregnanciesan-observational-cohort-study-based-on-propensity-score-matching 
Figure 1. Enrollment and follow-up flow diagram

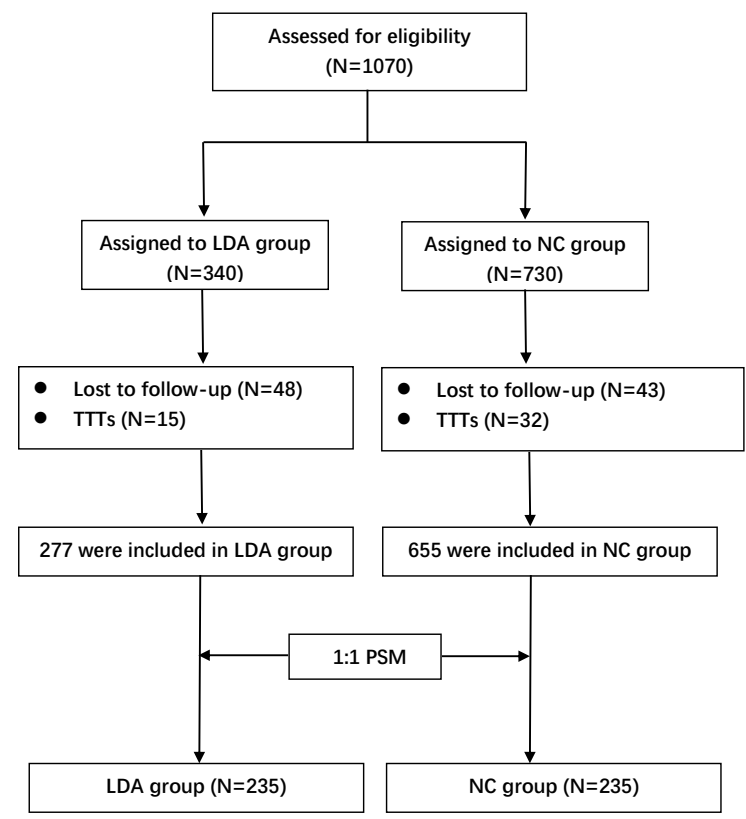

